\title{
Design and Construction of A Laser-Based Respiratory Gating System For Implementation of Deep Inspiration Breathe Hold Technique in Radiotherapy Clinics
}

\begin{abstract}
Background: Deep inspiration breath-hold (DIBH) is known as a radiotherapy method for the treatment of patients with left-sided breast cancer. In this method, patient is under exposure only while he/she is at the end of a deep inspiration cycle and holds his/her breath. In this situation, the volume of the lung tissue is enhanced and the heart tissue is pushed away from the treating breast. Therefore, heart dose of these patients, using DIBH, experiences a considerable decline compared to free breathing treatment. There are a few commercialized systems for implementation of DIBH in invasive or noninvasive manners. Methods: We present a novel constructed noninvasive DIBH device relied on a manufacturing near-field laser distance meter. This in-house constructed system is composed of a CD22-100AM122 laser sensor combined with a data acquisition system for monitoring the breathing curve. Qt Creator (a cross-platform JavaScript, QML, and C++-integrated development environment that is part of the SDK for development of the Qt Graphical User Interface application framework) and Keil MDK-ARM (a programming software where users can write in $\mathrm{C}$ and $\mathrm{C}++$ and assemble for ARM-based microcontrollers) are used for composing computer and microcontroller programs, respectively. Results: This system could be mounted in treatment or computed tomography (CT) room at suitable cost; it is also easy to use and needs a little training for personnel and patients. The system can assess the location of chest wall or abdomen in real time with high precision and frequency. The performance of CD22-100AM122 demonstrates promise for respiratory monitoring for its fast sampling rate as well as high precision. It can also deliver reasonable spatial and temporal accuracy. The patient observes his/her breathing waveform through a 7" $1024 \times 600$ liquid crystal display and gets some instructions during treatment and CT sessions by an exploited algorithm called "interaction scenario" in this study. The system is also noninvasive and well sustainable for patients. Conclusions: The constructed system has true real-time operation and is rapid enough for delivering clear contiguous monitoring. In addition, in this system, we have provided an interaction scenario option between patient and CT or Linac operator. In addition, the constructed system has the capability of sending triggers for turning on and off CT or Linac facilities. In this concern, the system has the superiority of combining a plenty of characteristics.
\end{abstract}

Keywords: Deep inspiration breath-hold, gated radiotherapy, respiratory-gated treatment, respiratory monitoring system, tumor tracking

\section{Introduction}

Deep inspiration breath-hold (DIBH) is a reliable technique for decreasing cardiopulmonary doses of patients who suffer from left-sided breast cancer (LSBC) in radiotherapy treatments. ${ }^{[1-3]}$ It is also supposed that this technique decreases the long-term risk of cardiac injuries. ${ }^{[4]}$ The significance of respiratory motion management $t^{[4]}$ has caused the

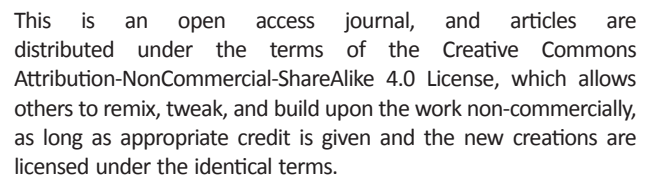

For reprints contact: reprints@medknow.com expansion of novel methods during the last decades, ${ }^{[5,6]}$ employing both invasive and noninvasive methods. There are various breathing management systems in the market such as Real-time Positioning Management ${ }^{\mathrm{TM}}$ (RPM) (Varian Medical System), Active Breathing Coordinator ${ }^{\mathrm{TM}}$, and AZ-733 V (Anzai Medical) which are available for obtaining respiratory cycle signal and performing respiratory gating techniques [Figure 1]..$^{[7,8]}$

\footnotetext{
How to cite this article: Naseri S, Farzaneh MJ, Momennezhad M, Salek R. Design and construction of a laser-based respiratory gating system for implementation of deep inspiration breathe hold technique in radiotherapy clinics. J Med Sign Sens 2018; XX:XX-XX.

Received: July, 2018. Accepted: August, 2018.
}

\section{Mohammad Javad Keikhai Farzaneh ${ }^{1}$, Shahrokh Nasseri ${ }^{1,2}$, Mehdi Momennezhad ${ }^{1,3}$, Roham Salek ${ }^{4}$}

${ }^{\prime}$ Department of Medical Physics, Faculty of Medicine, ${ }^{2}$ Medical Physics Research Center, ${ }^{3}$ Nuclear Medicine Research Center, ${ }^{4}$ Department of Radiation Oncology, Imam Reza Hospital, Mashhad University of Medical Sciences, Mashhad, Iran

Address for correspondence: Dr. Shahrokh Naseri,

Department of Medical Physics, Faculty of Medicine, Mashhad University of Medical Sciences, Pardis-e-daneshgah, Vakil Abad Blvd, Mashhad, Iran

E-mail: naserish@mums.ac.ir

Website: www.jmss.mui.ac.ir DOI: $10.4103 /$ jmss.JMSS_35_18 


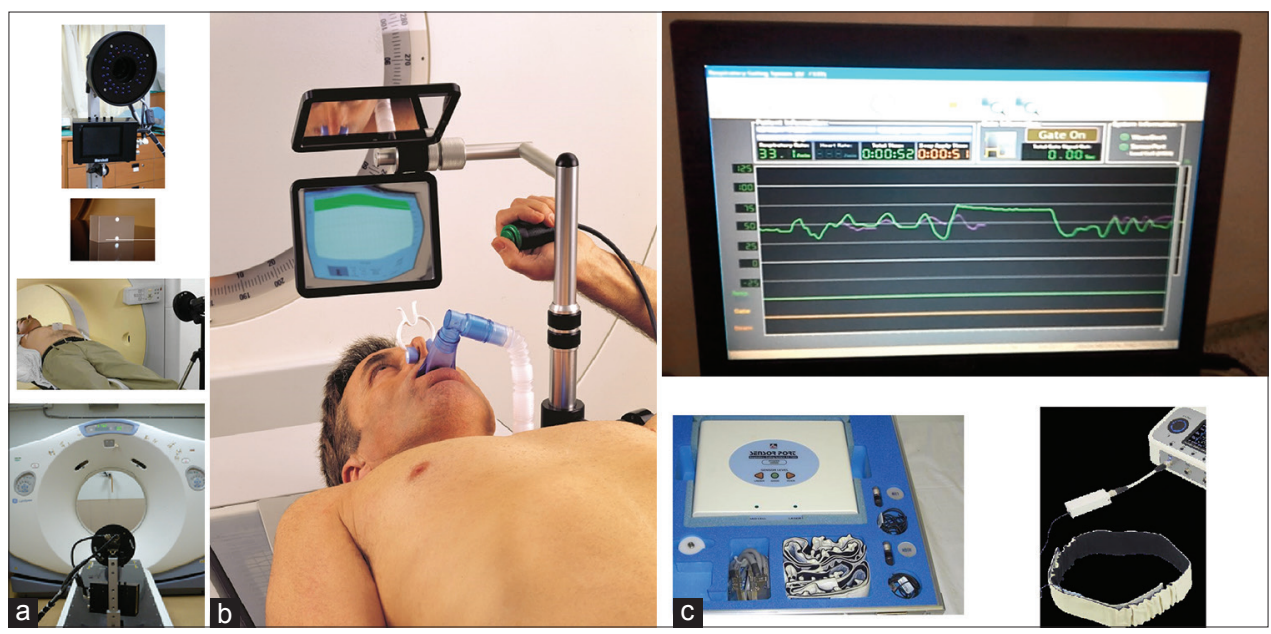

Figure 1: Some of the commercialized respiratory gating systems; (a) Real-time Position Management ${ }^{\mathrm{TM}}$ (Varian Medical System), (b) Active Breathing Coordinator $^{\mathrm{TM}}$, and (c) AZ-733 V (Anzai Medical)

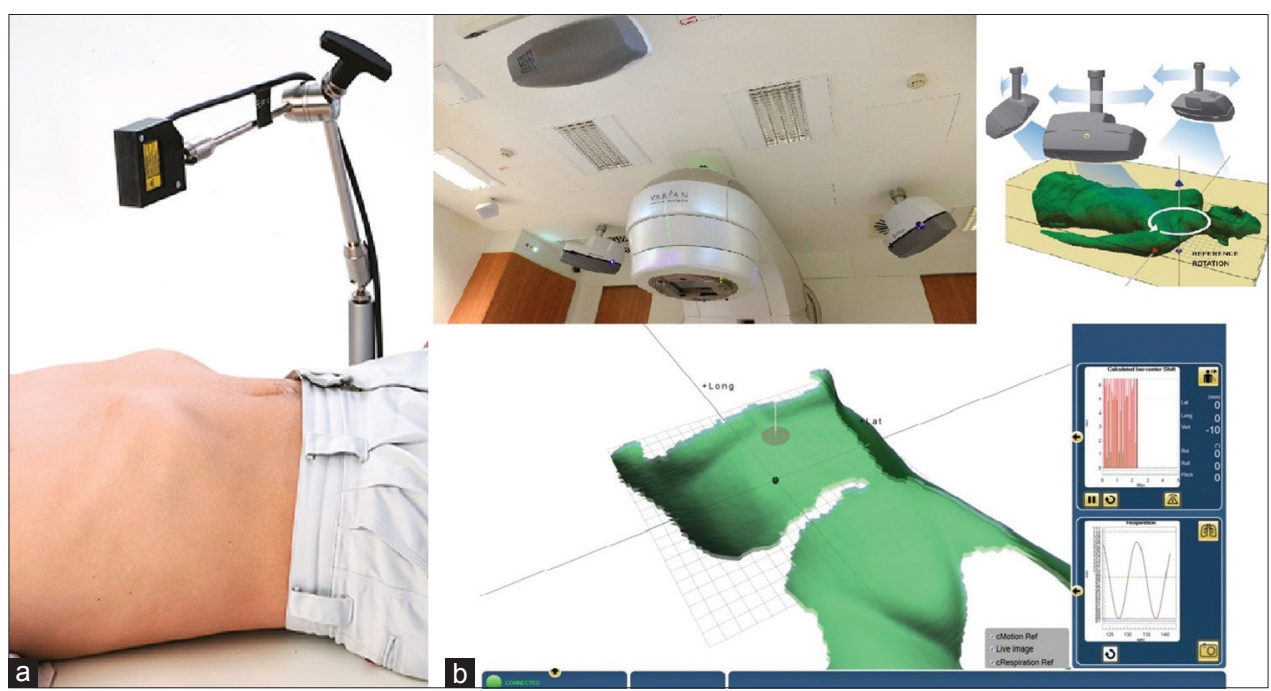

Figure 2: (a) Laser respiratory gating system of Anzai (Anzai Medical, Tokyo, Japan) and (b) Catalyst (C-RAD Positioning, Uppsala, Sweden) respiratory gating system

The RPM works relying on the remote monitoring of a box that contains markers of infrared, placed upon the xiphoid process of patient. When using RPM, it is probable that the position of the box, on the patient's body, differs from fraction to fraction. In addition, box placement upon the surface body of the patient can increase the skin dose of the patient due to the buildup effect if the box is placed within the field borders [Figure 1a]. ${ }^{[9]}$

Active breathing control $(\mathrm{ABC})$ is also another technique for implementation of breath-holding [Figure 1b]. ${ }^{[10-13]}$ This technique has been developed in William Beaumont Hospital and is money oriented by Elekta Company under the title of "Active Breathing Coordinator ${ }^{\mathrm{TM}}$." Another device with the same abilities named " $\mathrm{V}_{\max }$ Spectra $20 \mathrm{C}$ " is commercialized by VLASYS Healthcare Incorporation. Active breath-holding systems can stop respiratory procedure in a predefined level, which are normally deep or average inhalations. In these systems, one spirometer transducer is attached to a tube and the tube is coupled to the patient's mouth while the patient's nose is blocked by a fastener and patient can only breathe via the spirometer. Then, the spirometer measures the air stream that enters into or exists from the patient's lungs and at the next step, this air stream turns to air volume for attaining the lung movements. ${ }^{[14,15]}$ In this system, a spirometer is linked to a laptop and the physician is capable of seeing the patient's breathing cycle on the monitor. For having a consistent implementation, the spirometer's valve gets closed automatically when the patient's breathing level enters the predefined gating window. The duration of breath-holding depends on the capability of the patients, usually between 15 and $20 \mathrm{~s}$, and could be performable easily by the patient to provide a repeatable breath-holding without any discomfort to the patient. For carrying out the DIBH technique by active breath-holding systems, the main concern is repeatability of breath-holding and the 
patient's ability to follow this technique. Fassi et al. ${ }^{[16]}$ have compared the sustainability and repeatability of the DIBH technique implementation monitored by spirometer, with the DIBH technique monitored with infrared optical tracking system. They reported that monitoring the patient's breathing cycle by spirometer cannot provide a repeatable DIBH status in a radiotherapy fraction.

Another respiratory gating system called AZ-733 V (Anzai Medical, Tokyo, Japan) uses a pressure sensor located within a stretchy belt [Figure 1c]. The belt could be wrapped on all sides of the patient's chest wall or abdomen. By patient's respiration, the applied pressure upon the sensor changes and this variation shows function of time in a graph. ${ }^{[1,17]}$ This system is less invasive but not always sustainable by patients. Anzai (Anzai Medical) has also newly commercialized a respiratory gating system which uses a laser sensor, but till now there is no published data about this system [Figure 2a]. ${ }^{[18]}$ Jensen et al. ${ }^{[19]}$ also reported a DIBH system relying on an industrial laser distance measurer. Their equipment can measure the position of the chest wall with high precision and frequency and due to noninvasiveness nature, it is well tolerated by patients. Complete noninvasive facilities, such as Catalyst (C-RAD Positioning, Uppsala, Sweden), project a pattern of light upon the patient's body and then scan it with a high-resolution charge-coupled device camera to generate a three-dimensional-reconstructed topology from the patient's body [Figure $2 \mathrm{~b}]^{\left[{ }^{[16,20]}\right.}$

In another method which is voluntary breathe-holding without respiratory monitoring, patient holds his/her breathe in a portion of respiratory period, voluntarily. For the time of breath-holding, the beam of radiation is on and patient is under exposure. For implementation of this technique, a control system is developed for the Varian C $\operatorname{series}^{[21,22]}$ accelerators that use customer minor (CMNR) interlock. The patient holds a switch in his/her hand, which is connected to a circuit of CMNR. When this switch is pressed by the patient, the CMNR interlock gets opened in the control console and permits the operator to turn on the Linac. When the button is pushed off, the CMNR interlock becomes energetic and turns off the beam. In this method, activation of CMNR interlock prevents from dose delivering to patient until the switch is pressed again. It should also be noted that although in this system both the operator and patient can turn the beam off, just the operator can turn the beam on. Another thing that should be considered is that this system is not available commercially. There is also some other systems such as infrared reflector systems, breathing stick, cine electronic imaging, leveling marks, magnets, and stretching belts, which have been used for the implementation of DIBH technique. ${ }^{[19,23-26]}$

According to studies, in deep inhalation and exhalation, the highest degree of repeatability happens in body posture. This issue along with other potential benefits that occur as a result of the lung volume enhancements has turned the DIBH to a more favorable technique in breath-holding. ${ }^{[27,28]}$

In this study, a new in-house constructed DIBH gating system is presented. This new designed system uses a near-field laser distance meter sensor for real-time motion monitoring of chest wall or abdomen while breathing. This new constructed in-house system is noninvasive, so there is no discomfort for patient. Furthermore, patient views the waveform of his/her chest or abdomen displacement, as a function of time, in a 7" liquid crystal display (LCD). This system can be installed in treatment or computed tomography (CT) room at a low cost and limited training is needed to its use. Our main goal in this research was to design and construct a new DIBH gating system with suitable performance and price. In this system, we have also used a novel algorithm for establishment of an interaction scenario between operator and patient. Therefore, in clinics that there are some limitations for connecting this in-house constructed DIBH gating system to the CT scanner or Linac, in order to synchronizing CT acquisition or treatment dose delivery with the patient's breathing cycle, this interaction scenario provides an excellent tool for performing repeatable and healthy DIBH technique.

\section{Materials and Methods}

NXP (founded by Philips Semiconductors, Incorporation.) LPC1788, which is an ARM Cortex-M3 microcontroller, was used for designing and constructing the system's electronic hardware [Figure 3a]. For this goal, an in-house electronic circuit was designed to cover breathing management system's requirements [Figure 3b].

Computer software and LPC1788 microcontroller programs were developed in Qt Creator and Keil MDK-ARM, respectively. This in-house respiratory management system utilizes a CD22-100AM122 laser distance measurer transducer.

CD22-100AM122 is a diffuse type of triangulation sensor. It uses a red laser diode with a wavelength of $650 \mathrm{~nm}$ as a transmitter for measuring absolute distances from reflecting and natural surfaces. In this sensor type, the laser beam emitted from the semiconductor laser is applied to the target and the light reflected from the target is gathered by the detector lens and collimated on the light detector element. While changing target-to-sensor distance, the angle of the reflected light, passing via the detector lens, changes and the light is focused at different positions on the light detector element [Figure 4]. As a consequence, the sensor creates a current relative to the position of the spot on the detector. Finally, by using this current amount, the distance of the target to sensor can be calculated by the 


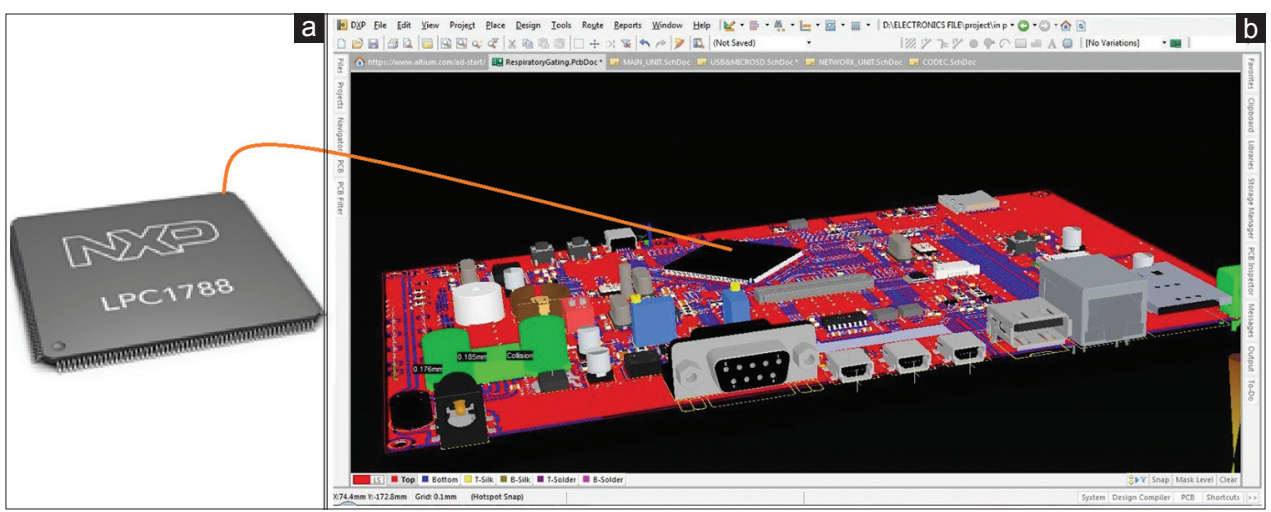

Figure 3: (a) LPC 1788 microcontroller. (b) Three-dimensional schematic of the constructed electronic circuit for the system

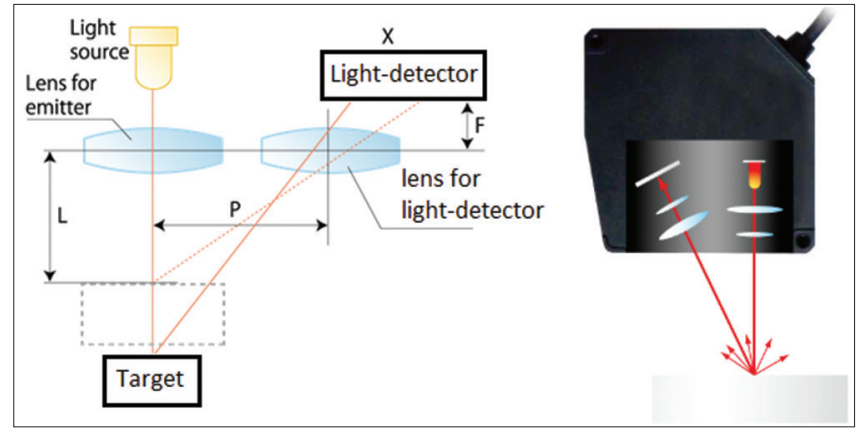

Figure 4: Basic operation principle of CD22-100AM122 laser sensor

following formula in the electronic processor section of the sensor:

$L=\frac{P \times F}{X}$

In this formula, " $L$ " is the distance of the sensor to target, $P$ is the distance between transmitter and receiver lenses, $F$ is the distance between light detector and detector lens, and $\mathrm{X}$ is the current of light detector.

The measurement range of the CD22-100AM122 laser sensor is $100 \pm 50 \mathrm{~mm}(100 \mathrm{~mm}$ is the center of range and $\pm 50 \mathrm{~mm}$ is the span of it). In normal operation, the sensor's repeatability and linearity are $20 \mu \mathrm{m}$ and $100 \mu \mathrm{m}$, respectively, but further characteristics which individualize this sensor among others are its onboard digital display and high sampling rate of $2000 \mathrm{~Hz}$.

The laser sensor, at a distance of $50-150 \mathrm{~mm}$ from the sensor location, provides a variable current between 4 and $20 \mathrm{~mA}$ at its output. At the next step, sensor output current is applied to a $100 \Omega$ resistance for generating an analog voltage between 0.4 and $2 \mathrm{~V}$. Subsequently, this analog voltage is applied to a 16-bit analog-to-digital convertor (ADC) called AD7705BR for digitization. The reference voltage of the ADC is $2.5 \mathrm{~V}$ and in this condition the ADC converter resolution is acquired at about $\frac{2.5}{2^{16}}=38 \mu \mathrm{V}$. This means that for every $38 \mu \mathrm{V}$ changes at the input of ADC, we have one unit changes at the ADC output. Afterward, these digital data transmit to the LPC1788 microcontroller for analysis.
Finally, the LPC 1788 is connected to a personal computer (PC) via a RS-232 serial interface and respiratory waveform showed on the PC monitor as well as patient's LCD [Figure 5].

\section{Installation}

As shown in Figure 6a, we have designed a laser box for locating the laser sensor within it. There is also a constructed box holder in order to adjust the laser box in various coordinates of the $\mathrm{CT}$ or treatment rooms. The laser box takes place upon the designed holder and the holder is installed on a fixed base, beside or upon the CT or treatment tables. The size of the laser box is $5 \mathrm{~cm} \times 5 \mathrm{~cm} \times 5 \mathrm{~cm}$ and its surfaces are scaled in 1-mm intervals. Therefore, we can adjust the laser box at an exact specified position relative to the isocenter of treatment or CT rooms. The position of the laser box can also be adjusted freely at both vertical and horizontal orientations through changing angles of the box holder arms [Figure 6b]. In addition, position and angle of the sensor relative to the patient body isocenter are constant in both CT and treatment rooms because after patient positioning as well as adjusting laser sensor on the patient body in CT room, technologists record the exact position of the center of the laser box relative to the CT simulation isocenter, in CT simulation sheet for future treatment session adjustments. For this goal, technologists change the position of the $\mathrm{CT}$ bed for locating the center of laser box at the isocenter of the CT room and record the changes of CT bed position, relative to the patient isocenter. At treatment sessions, technologists use these data to adjust the laser box at an exact position relative to the patient isocenter, again. In this way, all adjustments are same in both CT simulation and treatment sessions and the position of the laser sensor in whole of the treatment procedure is same and similar to CT simulation session adjustment. In brief, isocenter of the treatment and CT rooms has the role of precautionary device for avoidance of any error while using this respiratory gating system. 
Control panel of the constructed respiratory gating system

In our constructed respiratory gating system, therapeutists can adjust the baseline and width of the gate window in the control panel of the system in the CT session as well as in each treatment session. The therapeutist determines the high level of the window by adjusting the value of gating upper level (GUL) and the low level of the window by adjusting the value of gate lower level (GLL) in the system's control panel. Therapeutist can also move the whole respiratory curve up or down on the monitor by changing the value of the baseline. In the control panel of this system, we have designed three distinct parts of "Operator Panel," "Patient Condition," and "Patient Observation." In "operator panel," we have "Start," "Stop," and "Ready to patient command" icons as well as an adjustable timer. There are "Pushed on the key," "Pushed off the key," and "Laser: out of gate" icons in "Patient condition" section of the system's control panel. Finally, in the "Patient observation" section, "Ray On," "Ray Off," and "command" could be appeared. Furthermore, a timer is visible in this section [Figure 7].

At our clinic, the operator of the CT scanner or Linac was needed to manually provoke the beam-on mode once the respiratory period comes into the window gap. Therefore, in order to avoid mistreatments, improve patient safety, and increase the precision and efficiency of the procedure, we have used an interaction scenario in this constructed gating

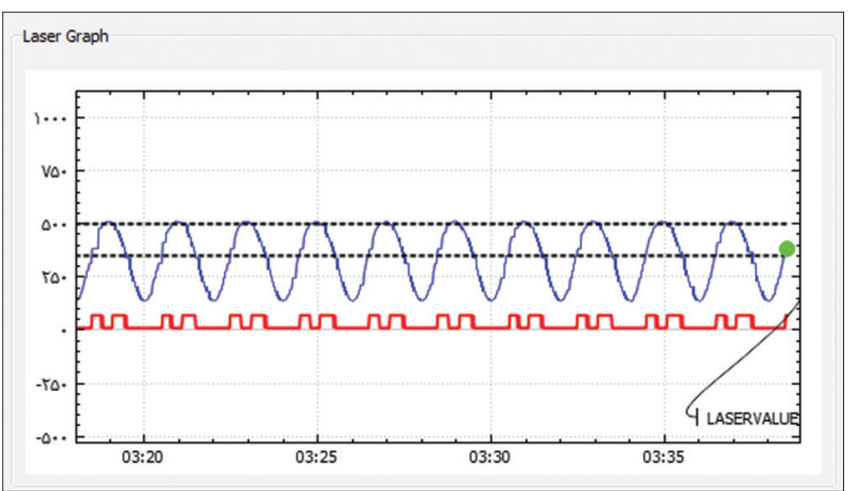

Figure 5: Waveform of the respiratory motion tracking test phantom plotted by the constructed gating system

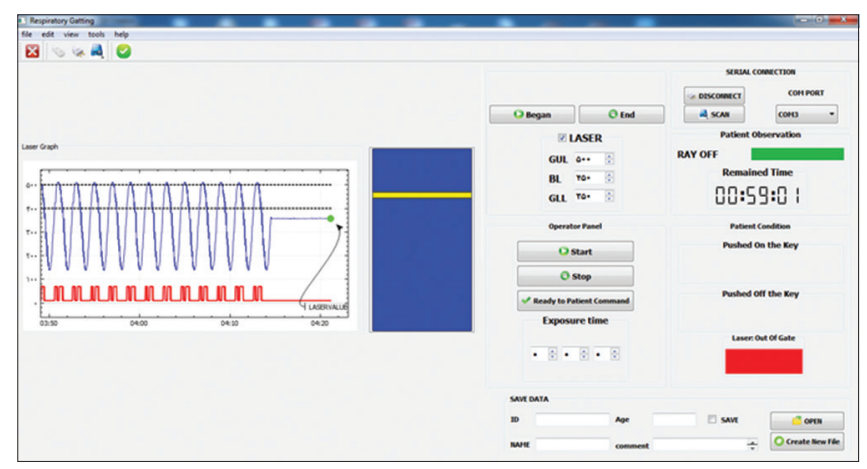

Figure 7: Control panel of the constructed respiratory gating system system. In this scenario, the respiratory cycle is shown, on a 7" LCD, to patients during the CT simulation as well as treatment dose delivery [Figure 8].

In the first step, the patient's LCD is turned on and a push button takes place into the hand of the patient. Then, an operator (standing behind the patient's couch) asks the patient for DIBH implementation. In this situation, GLL, GUL, and baseline are set by the operator and recorded in the patient's CT simulation sheet for future adjustments at treatment sessions. After patient's setup and gating system adjustments, the operator goes to the control room of the CT (or Linac) to initiate the imaging (or treatment) procedure using the gating system. At this time, the operator clicks on the "Ready to patient command" icon in the "Operator panel" of the gating system control panel. By doing this, the "Command" icon on the patient's LCD screen as well as the "Patient observation" section of the constructed gating system control panel turns on simultaneously. By turning on the "Command" icon on the patient's LCD, the patient realizes that she/he has to do some normal breathing and then perform DIBH technique (take a deep breath and keep

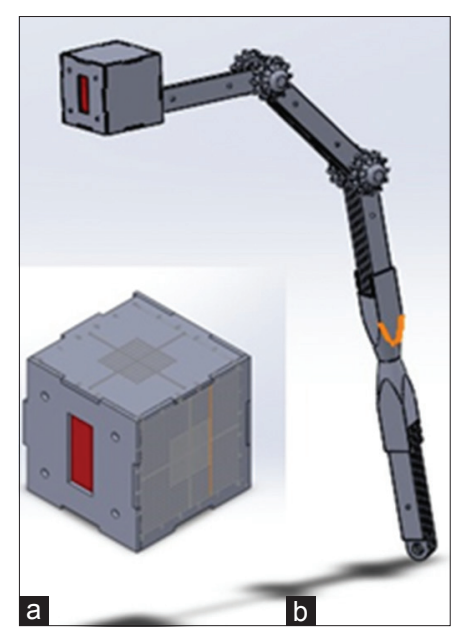

Figure 6: (a) Constructed laser box and (b) laser box holder

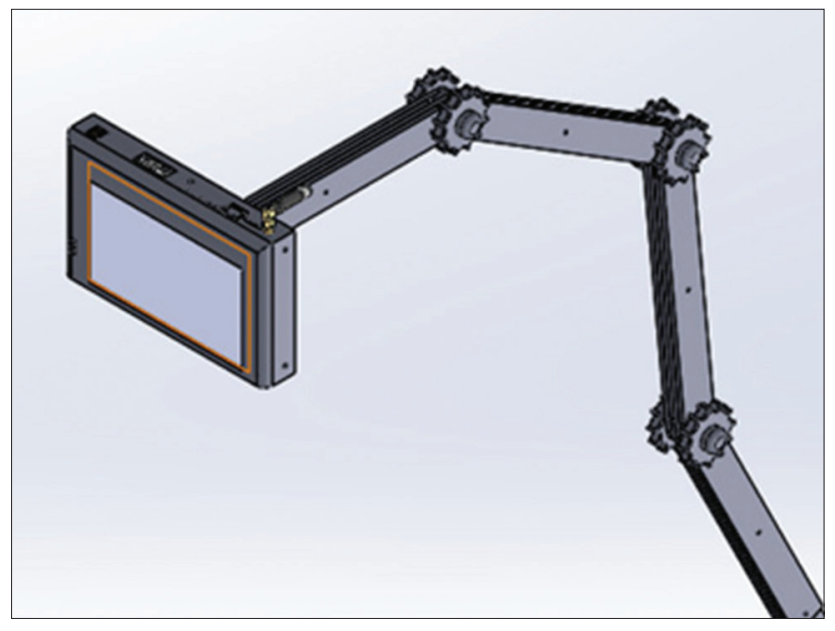

Figure 8: Patient's liquid crystal display upon its holder 
her/his breathing signal inside the window). The patient also knows that while performing DIBH technique, she/he must press down the push button which is located on her/his hand. Right after pressing down the push button by the patient, the "Pushed on the key" icon is turned on in "Patient condition" section of the system's control panel and the operator realizes that the patient is ready for starting the imaging (or treatment) procedure. In this condition, if the patient's respiratory signal is within the window, the operator clicks on the "Start" icon in the control panel of the DIBH gating system. By clicking on the "Start" icon by the operator, the "Ray on" icon turns on upon the patient's LCD as well as "Patient observation" sections of the system's control panel. By seeing "Ray on" icon upon the LCD, the patient realizes that CT imaging (or dose delivery) procedure is begun and she/he should continue breath-holding. The operator then presses the start button of the CT (or accelerator) to begin the CT imaging (or treatment delivery) procedure. In this situation, two statuses can occur; the first one is that the patient leaves the push button and the second is that, for any reason, respiratory level of the patient exits from DIBH window. In both of these situations, the CT imaging (or treatment delivery) must be stopped. At the first case, when the patient leaves the push button, the "Pushed off the key" icon turns on in "Patient Condition" section and the operator finds out that the patient can no longer continue DIBH implementation. Under these circumstances, the operator first stops the CT imaging (or treatment delivery) procedure and then clicks on the "Stop" icon in the DIBH system's control panel. Right after clicking on the "Stop" icon, the "Ray On" icon turns off and "Ray off" icon turns on upon the patient's LCD as well as "Patient Observation" sections of the operator. In this situation, the patient realizes that she/he can release her/his breath-holding and breathe normally. In the second case, when the signal brings out from the predefined window, "Laser: out of gate" icon turns on upon the operator control panel and the operator realizes that she/he must stop the CT imaging (or treatment delivery) procedure. For this goal, the operator first stops the process of CT imaging (or treatment delivery) and then clicks on the "Stop" icon of the DIBH system's control panel. Again, right after clicking on the "Stop" icon, the "Ray On" icon turns off and "Ray off" icon turns on upon the patient's LCD as well as operator's control panel and the patient realizes that she/he can release her/his breath-holding and can breathe normally. Then, the operator permits the patient for normal breathing in about 5-10 $\mathrm{s}$ and then clicks on "Ready to patient command" icon for continuing CT imaging (or treatment delivery) procedure with DIBH technique. This process repeats frequently till completing CT imaging (or treatment delivery).

Flowcharts depicted in Figures 9 and 10 are developed for working with interaction scenario of the in-house constructed gating system, in CT simulation and treatment rooms, respectively. As mentioned, our constructed

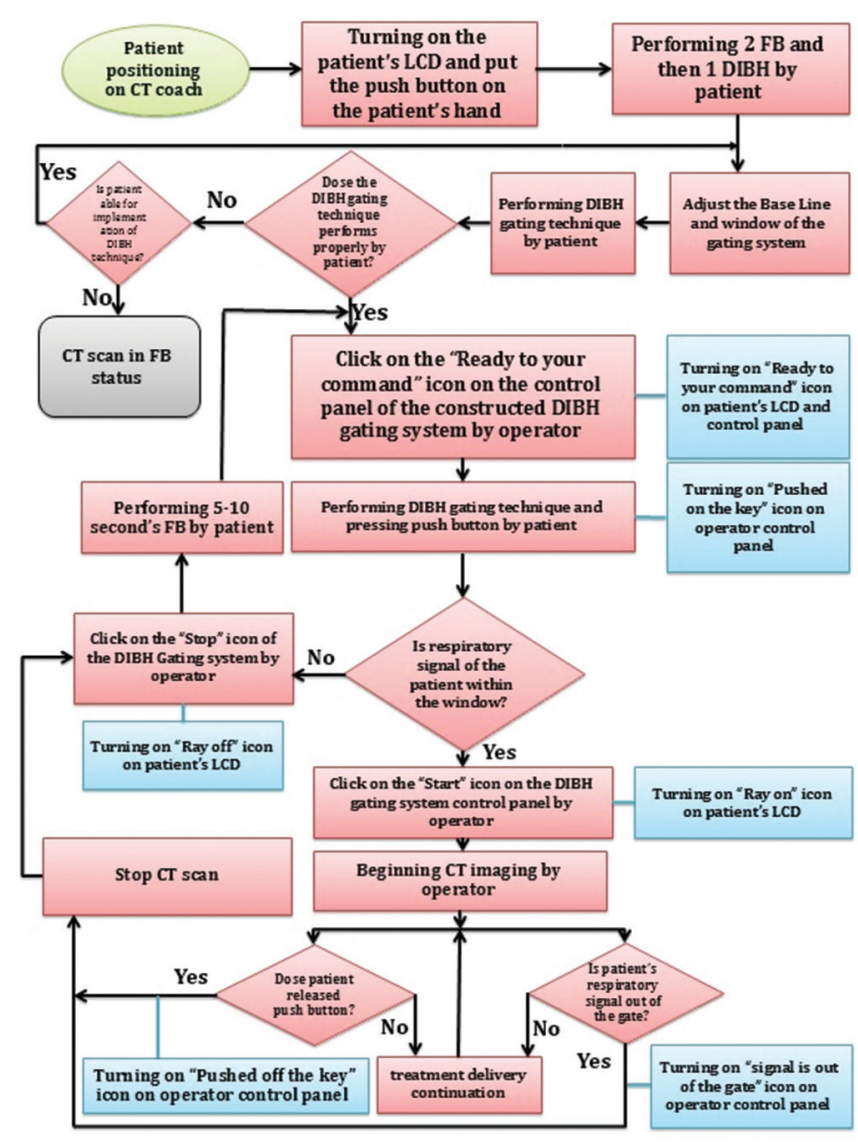

Figure 9: Flowchart of the deep inspiration breath hold technique implementation by the in-house constructed gating system in computed tomography simulation room

respiratory gating system can perform gating through direct connection to the CT or Linac facilities. Unfortunately, at this time, CT and Linac facilities of our clinic are not active for implementation of respiratory gating, but we have equipped the constructed gating system to this feature for future opportunities.

For attaining this goal, we have assigned a discrete port of the device. This port is in high-level status (level 1) while the respiratory signal is within the gating window and falls to the low level (level 0) right after getting out the signal from the predefined gate. By applying this signal to the CT or Linac, we can perform respiratory gating techniques on patients. For this aim, we should only accommodate the output signal level of the constructed gating system with the required input signal of the employed CT or Linac. Finally, by applying the signal of this dedicated port to CT or Linac, radiation beam of these facilities will be turned on and off according to the command of the constructed gating system synchronously.

Quality control of the in-house constructed respiratory gating system

Our respiratory gating system is invented specially for implementation of amplitude gating, and its quality control 
method is basically similar to that of the Varian RPM quality control procedure. For this, we have designed and constructed a respiratory motion tracking test phantom, similar to the Varian RPM test phantom, for simulation of respiratory amplitude and cycle [Figure 11]. The rotary motor motion of this phantom converts into a vertical motion, which is detectable by the in-house constructed respiratory gating system. By plotting these amplitude changes against the time axis, a curve will be formed which is similar to a sine wave in appearance.

For implementation of quality control procedure, we have adjusted the respiratory motion tracking test phantom for producing waveforms with $2-\mathrm{cm}$ amplitude and frequency of $0.5 \mathrm{~Hz}$. The laser spot point with size of $600 \mu \mathrm{m} \times 700 \mu \mathrm{m}$ (at a distance of $10 \mathrm{~cm}$ away from the sensor position) was focused on the vertical axis of the plastic circle of test phantom. By this way, the laser can detect the amplitude changes of the plastic circle in real time and give us the sinusoidal waveform at output of the device.

A step motor (MINEBEA, 17PM-K102, Thailand) shaft is connected to the plastic circle at the point which is $2 \mathrm{~cm}$ out of the circle center. The distance of 72 points, at the circle circumference, from the point which is $2 \mathrm{~cm}$

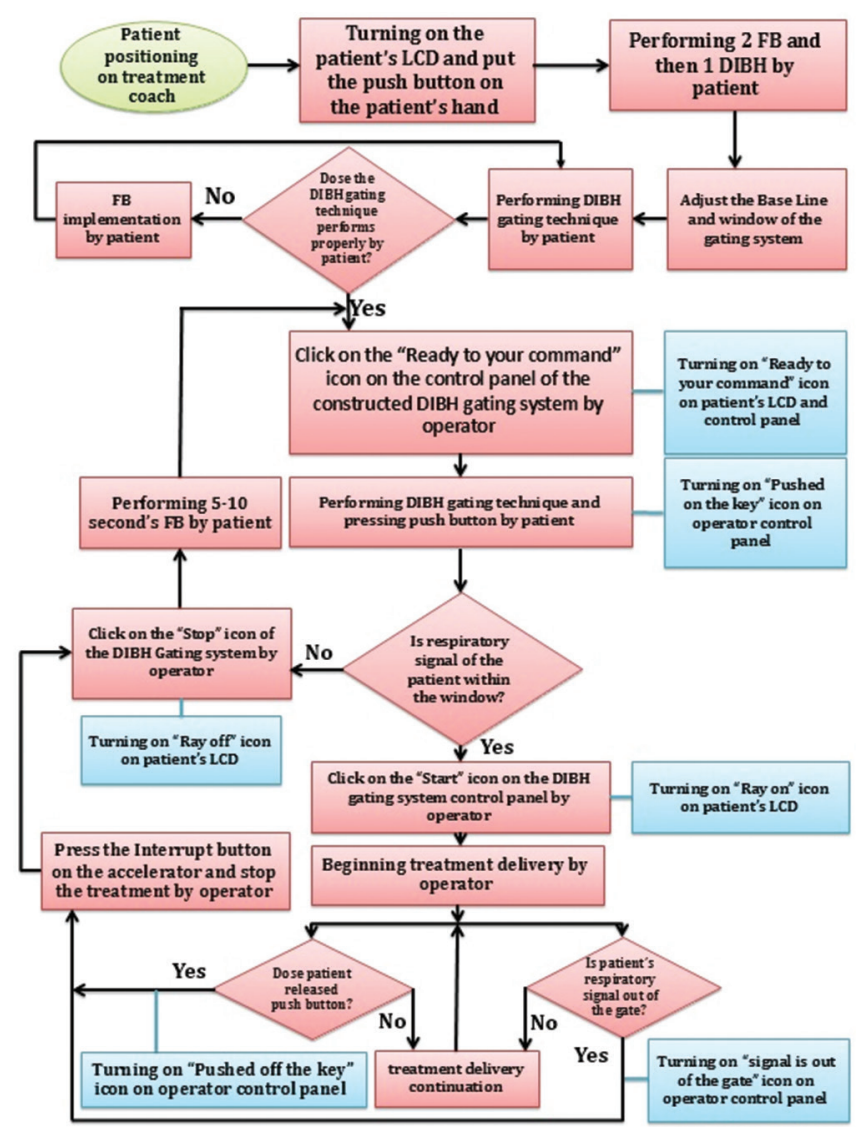

Figure 10: Flowchart of the deep inspiration breath-hold technique implementation by the in-house constructed gating system in treatment delivery room out of the circle center are written on the plastic circle [Figure 12]. Therefore, the eccentricity of these points is well recognizable at every time, While plastic circle is rotating.

CD22-100AM122 laser sensor has a measurement range of $10 \mathrm{~cm}$ and its neutral location (relative zero) is adjusted in the middle of this range which is $10 \mathrm{~cm}$ away from the sensor's position. Therefore, the laser sensor is located $10 \mathrm{~cm}$ away from the revolving circle and targeted on a specified point upon the plastic circle circumference [Figure 11].

\section{Results}

\section{Detection linearity}

The linearity of the sensor and computer reading over $40 \mathrm{~mm}$ span was very good and R2 acquired 1.000 and 0.999 for laser and computer readings, respectively. Figures 12 and 13 show linearity of the sensor and computer readings.

\section{Detection reproducibility}

The reproducibility of the in-house constructed system was also evaluated via digital numbers utilized for plotting respiratory waveform. For this purpose, we have adjusted the circumference of the revolving circle of the test phantom at an exact distance from the laser sensor for 10 times and noted its related digital numbers transmitted to the computer, by a gating system. This procedure was done for ten different distances within the range of $80.00-120.00 \mathrm{~mm}$ from the sensor position. Mean and standard deviation of digital numbers transmitted to the computer are shown in Table 1.

\section{Detection accuracy}

The detection accuracy of the sensor was also obtained by using Bias equation from the following equivalence.

Bias $=($ measured value - actual value $)$

In this regard, the average, maximum, and minimum differences between the actual value and measured value by the sensor, over the range of $80.00-120.00 \mathrm{~mm}$, were $0.08,0.60$, and -0.65 , respectively.

\section{Discussion}

The in-house constructed respiratory gating system was primarily developed as a stand-alone DIBH gating system;

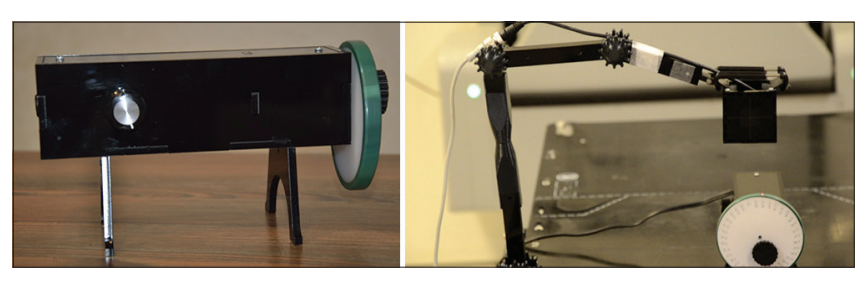

Figure 11: In-house designed and constructed respiratory motion tracking test phantom 


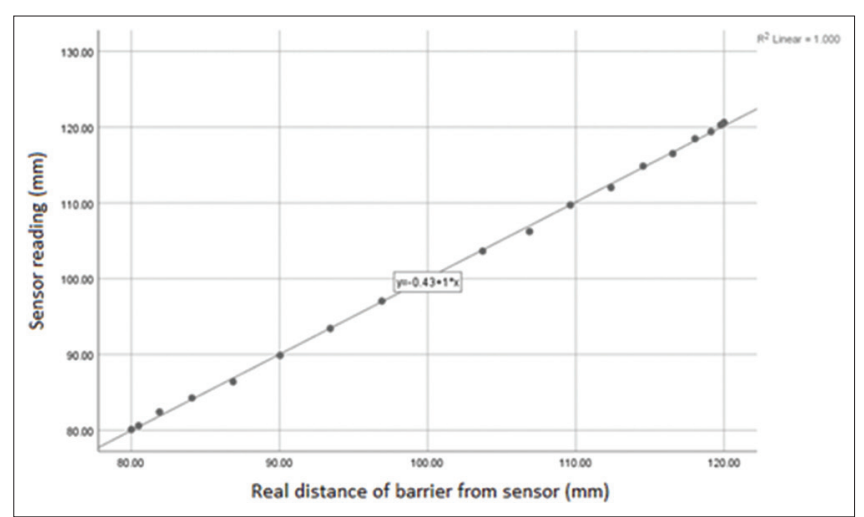

Figure 12: Linearity of sensor reading compared to the actual value over $40.00 \mathrm{~mm}$ span $\left(R^{2}=1.000\right)$

\begin{tabular}{lc}
\hline $\begin{array}{c}\text { Table 1: Mean and standard deviation of digital } \\
\text { numbers transmitted to the computer for ten different } \\
\text { distances from the sensor position }\end{array}$ \\
\hline Distance number & Mean \pm SD \\
\hline 1 & $511.20 \pm 0.79$ \\
2 & $382.00 \pm 0.67$ \\
3 & $395.30 \pm 0.95$ \\
4 & $299.90 \pm 0.57$ \\
5 & $294.70 \pm 0.82$ \\
6 & $260.90 \pm 0.88$ \\
7 & $243.40 \pm 0.84$ \\
8 & $194.20 \pm 0.63$ \\
9 & $200.70 \pm 0.67$ \\
10 & $138.10 \pm 0.57$ \\
\hline
\end{tabular}

SD - Standard deviation

however, it is now upgraded for the implementation of respiratory gating techniques. The performance of the system is also assessed against the in-house constructed respiratory motion tracking test phantom. We found a very good performance for the constructed gating system. We assessed the reproducibility of the system. Reproducibility was very good over the whole 40 -mm sinusoidal range of circle plastic displacement. This reproducibility shows the performance of the system for monitoring respiratory motions. One of the most significant advantages of the constructed respiratory gating system is its high signal-to-noise ratio (SNR) in the detection of respiratory waveforms. This in-house constructed laser respiratory gating system exhibits a noise level of about $0.76 \mathrm{mV}$, while the magnitudes of the indicated breathing curves (for 1-cm displacement amplitude) were bigger than $158 \mathrm{mV}$, corresponding to a SNR of 207 which is greatly higher than 20. Furthermore, those measurement outcomes can be indicated both quantitatively and qualitatively on the computer.

By using this in-house gating system, reproducibility can be enhanced by making minute alignment to the setup on the $\mathrm{CT}$ or treatment bed, body motions during treatment

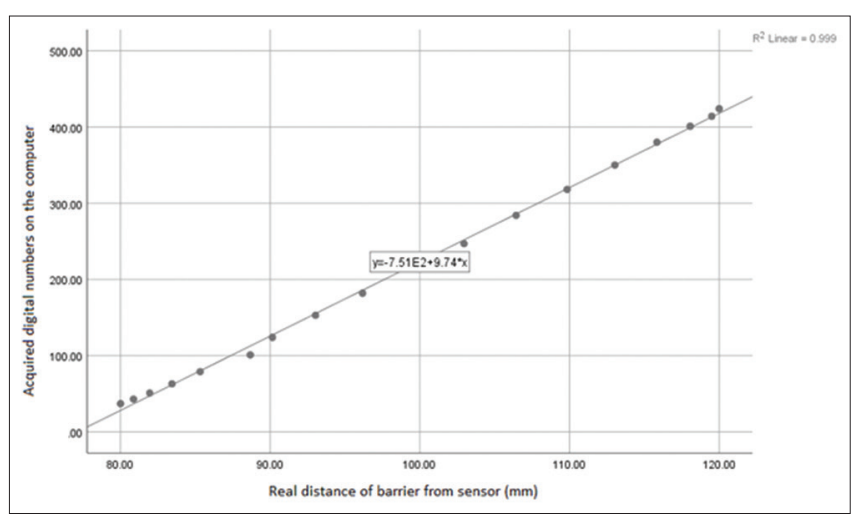

Figure 13: Linearity of computer reading compared to the actual value over $40.00 \mathrm{~mm}$ span $\left(R^{2}=0.999\right)$

and simulation can be tracked, and physical shifts during the CT and radiotherapy periods can be discovered. As a result, this gating system can operate as an assistive system to improve irradiation accuracy and precision during $\mathrm{CT}$ or treatment procedures.

This in-house constructed respiratory gating system is presently in clinical testing as a stand-alone system. Currently, we have focused on this system as a DIBH system for treatment planning and delivery of patients who suffer from breast cancer. Nevertheless, the system can also be utilized as an autonomous respiratory gating system. This gating system can measure the dislocation of the chest wall or abdomen with high frequency and precision in real time.

On the whole, this system can be used as a stand-alone system for implementation of DIBH and gating techniques, and radiation treatment outcome can increase by using it. Last but not the least, the most significant characteristics of this system are its noninvasiveness, clarity, and its interaction scenario option. Furthermore, the cost of this gating system is much lower than that of other commercially accessible respiratory management systems. The entire cost of this constructed respiratory gating system was $\$ 3000$ and this charge forms $<8 \%$ of the whole price of other commercially accessible respiratory gating systems. It should also be considered that implementation of the DIBH technique is not as convenience as free breathing (FB) status for patients, but its performance reduces the residual motion of the tumor to about $10 \%$, which is so significant compared to FB gating status with residual motion of $40 \%{ }^{[29]}$ Furthermore, studies have shown that exhibition of the respiratory signal to the patient during DIBH implementation is very helpful for attaining a repeatable level of DIBH. ${ }^{[7]}$ Therefore, in this in-house system, a visual feedback LCD was used for showing the respiratory signal to patient in real time. As a final deduction, implementation of more flawless therapeutic methods provides higher care for patients and as a result for the human society. In this regard, the constructed respiratory gating system in this study provides implementation of respiratory gating techniques in an easy and quick manner. 


\section{Financial support and sponsorship}

The authors thank the Vice-Chancellor for Research of Mashhad University of Medical Sciences, Mashhad, Iran for financial support (protocol code: 951544). In addition, the authors gratefully appreciate the cooperation of Nuclear Medicine Research Center in this study.

\section{Conflicts of interest}

There are no conflicts of interest.

\section{References}

1. Korreman SS, Pedersen AN, Nøttrup TJ, Specht L, Nyström H. Breathing adapted radiotherapy for breast cancer: Comparison of free breathing gating with the breath-hold technique. Radiother Oncol 2005;76:311-8.

2. Moran JM, Ben-David MA, Marsh RB, Balter JM, Griffith KA, Hayman JA, et al. Accelerated partial breast irradiation: What is dosimetric effect of advanced technology approaches? Int J Radiat Oncol Biol Phys 2009;75:294-301.

3. Nissen HD, Appelt AL. Improved heart, lung and target dose with deep inspiration breath hold in a large clinical series of breast cancer patients. Radiother Oncol 2013;106:28-32.

4. Keall PJ, Mageras GS, Balter JM, Emery RS, Forster KM, Jiang SB, et al. The management of respiratory motion in radiation oncology report of AAPM task group 76. Med Phys 2006;33:3874-900.

5. Low DA, Nystrom M, Kalinin E, Parikh P, Dempsey JF, Bradley JD, et al. A method for the reconstruction of four-dimensional synchronized CT scans acquired during free breathing. Med Phys 2003;30:1254-63.

6. Ford EC, Mageras GS, Yorke E, Ling CC. Respiration-correlated spiral CT: A method of measuring respiratory-induced anatomic motion for radiation treatment planning. Med Phys 2003;30:88-97.

7. Kini VR, Vedam SS, Keall PJ, Patil S, Chen C, Mohan R, et al. Patient training in respiratory-gated radiotherapy. Med Dosim 2003;28:7-11.

8. Otani Y, Fukuda I, Tsukamoto N, Kumazaki Y, Sekine H, Imabayashi $\mathrm{E}$, et al. A comparison of the respiratory signals acquired by different respiratory monitoring systems used in respiratory gated radiotherapy. Med Phys 2010;37:6178-86.

9. Pedersen AN, Korreman S, Nyström H, Specht L. Breathing adapted radiotherapy of breast cancer: Reduction of cardiac and pulmonary doses using voluntary inspiration breath-hold. Radiother Oncol 2004;72:53-60.

10. Remouchamps VM, Vicini FA, Sharpe MB, Kestin LL, Martinez AA, Wong JW, et al. Significant reductions in heart and lung doses using deep inspiration breath hold with active breathing control and intensity-modulated radiation therapy for patients treated with locoregional breast irradiation. Int J Radiat Oncol Biol Phys 2003;55:392-406.

11. van der Laan HP, Dolsma WV, van 't Veld AA, Bijl HP, Langendijk JA. Comparison of normal tissue dose with three-dimensional conformal techniques for breast cancer irradiation including the internal mammary nodes. Int $\mathrm{J}$ Radiat Oncol Biol Phys 2005;63:1522-30.

12. Rochet N, Drake JI, Harrington K, Wolfgang JA, Napolitano B, Sadek BT, et al. Deep inspiration breath-hold technique in left-sided breast cancer radiation therapy: Evaluating cardiac contact distance as a predictor of cardiac exposure for patient selection. Pract Radiat Oncol 2015;5:e127-34.

13. Mah D, Hanley J, Rosenzweig KE, Yorke E, Braban L, Ling CC, et al. Technical aspects of the deep inspiration breath-hold technique in the treatment of thoracic cancer. Int J Radiat Oncol Biol Phys 2000;48:1175-85.

14. Nehmeh SA, Erdi YE. Respiratory motion in positron emission tomography/computed tomography: A review. Semin Nucl Med 2008;38:167-76.

15. Zhang T, Keller H, O'Brien MJ, Mackie TR, Paliwal B. Application of the spirometer in respiratory gated radiotherapy. Med Phys 2003;30:3165-71.

16. Fassi A, Ivaldi GB, Meaglia I, Porcu P, Fatis PT, Liotta M, et al. Reproducibility of the external surface position in left-breast DIBH radiotherapy with spirometer-based monitoring. Journal of applied clinical medical physics. 2014;15(1):130-40.

17. Li XA, Stepaniak C, Gore E. Technical and dosimetric aspects of respiratory gating using a pressure-sensor motion monitoring system. Med Phys 2006;33:145-54.

18. AnzaiMedical. Available from: http://www.anzai-med.co.jp/en/ product/item/az733vi/index.html. [Last accessed on $2018 \mathrm{Jul} 22$ ].

19. Jensen CA, Skottner N, Frengen J, Lund JÅ. Development of a deep inspiration breath-hold system for radiotherapy utilizing a laser distance measurer. Journal of applied clinical medical physics. 2017;18(1):260-4.

20. Pallotta S, Marrazzo L, Ceroti M, Silli P, Bucciolini M. A phantom evaluation of sentinel( $\left.{ }^{\mathrm{TM}}\right)$, a commercial laser/ camera surface imaging system for patient setup verification in radiotherapy. Med Phys 2012;39:706-12.

21. Kim DJ, Murray BR, Halperin R, Roa WH. Held-breath self-gating technique for radiotherapy of non-small-cell lung cancer: A feasibility study. Int J Radiat Oncol Biol Phys 2001;49:43-9.

22. Barnes EA, Murray BR, Robinson DM, Underwood LJ, Hanson J, Roa WH, et al. Dosimetric evaluation of lung tumor immobilization using breath hold at deep inspiration. Int J Radiat Oncol Biol Phys 2001;50:1091-8.

23. Macrie BD, Donnelly ED, Hayes JP, Gopalakrishnan M, Philip RT, Reczek J, et al. A cost-effective technique for cardiac sparing with deep inspiration-breath hold (DIBH). Phys Med 2015;31:733-7.

24. Brouwers PJ, Lustberg $\mathrm{T}$, Borger $\mathrm{JH}$, van Baardwijk AA, Jager JJ, Murrer LH, et al. Set-up verification and 2-dimensional electronic portal imaging device dosimetry during breath hold compared with free breathing in breast cancer radiation therapy. Pract Radiat Oncol 2015;5:e135-41.

25. Kinoshita R, Shimizu S, Taguchi H, Katoh N, Fujino M, Onimaru $\mathrm{R}$, et al. Three-dimensional intrafractional motion of breast during tangential breast irradiation monitored with high-sampling frequency using a real-time tumor-tracking radiotherapy system. Int J Radiat Oncol Biol Phys 2008;70:931-4.

26. Remouchamps VM, Huyskens DP, Mertens I, Destine M, Van Esch A, Salamon E, et al. The use of magnetic sensors to monitor moderate deep inspiration breath hold during breast irradiation with dynamic MLC compensators. Radiother Oncol 2007;82:341-8.

27. Stromberg JS, Sharpe MB, Kim LH, Kini VR, Jaffray DA, Martinez AA, et al. Active breathing control (ABC) for hodgkin's disease: Reduction in normal tissue irradiation with deep inspiration and implications for treatment. Int $\mathrm{J}$ Radiat Oncol Biol Phys 2000;48:797-806.

28. Rosenzweig KE, Hanley J, Mah D, Mageras G, Hunt M, Toner S, et al. The deep inspiration breath-hold technique in the treatment of inoperable non-small-cell lung cancer. Int J Radiat Oncol Biol Phys 2000;48:81-7.

29. Yewondwossen M, Denissova S, Andrew J, Hale M, Murphy C, Purcell S. Gated Deep Inspiration Breath-hold Radiation Therapy Using a Linear Position Transducer. Medical Physics. 2003;30(6):1512. 


\section{BIOGRAPHIES}

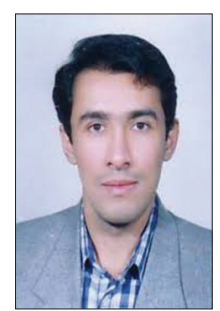

Shahrokh Naseri obtained his Ph.D in Medical Physics from Mashhad University of Medical Science in 2008. He is Assistant professor at Medical Physics Division in Mashhad University of Medical Sciences. His research interests include Radiotherapy and Image Processing.

Email: naserish@mums.ac.ir

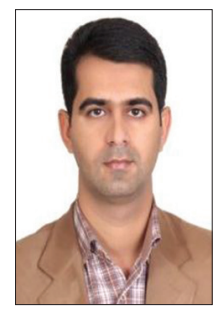

Mohammad Javad Keikhai Farzaneh obtained his Ph.D in Medical Physics from Mashhad University of Medical Science in 2018. He is Lecturer and Faculty Member of Medical Physics Division in Zahedan University of Medical Sciences. His research interests include Radiotherapy, Microcontroller Programming and Radiation Protection.

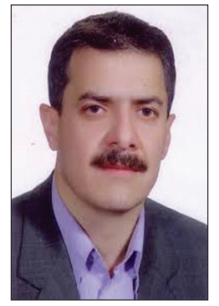

Mehdi Momennezhad obtained his Ph.D in Medical Physics from Mashhad University of Medical Science in 2006. He is Associate professor at Medical Physics Division in Mashhad University of Medical Sciences. His research interests include Radiotherapy, Nuclear Medicine, Dosimetry and Simulation.

Email: momennezhadm@mums.ac.ir

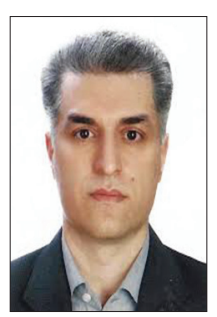

Roham Salek has finished Radiation oncology residency training in Mashhad University of Medical Science in 1997. He is Associate professor at Oncology Division in Mashhad University of Medical Sciences. His research interests include radiation therapy, chemotherapy, hormone therapy, and palliative care.

Email:salekr@mums.ac.ir 